\title{
ON PARAMETER ESTIMATION IN THE BASS MODEL BY NONLINEAR LEAST SQUARES FITTING THE ADOPTION CURVE
}

\author{
DARIJA MARKOVIĆ, DRAGAN JUKIĆ \\ Department of Mathematics \\ University of Osijek, Trg Ljudevita Gaja 6, HR-31 000 Osijek, Croatia \\ e-mail: \{darija, jukicd\}@mathos.hr
}

\begin{abstract}
The Bass model is one of the most well-known and widely used first-purchase diffusion models in marketing research. Estimation of its parameters has been approached in the literature by various techniques. In this paper, we consider the parameter estimation approach for the Bass model based on nonlinear weighted least squares fitting of its derivative known as the adoption curve. We show that it is possible that the least squares estimate does not exist. As a main result, two theorems on the existence of the least squares estimate are obtained, as well as their generalization in the $l_{s}$ norm $(1 \leq$ $s<\infty)$. One of them gives necessary and sufficient conditions which guarantee the existence of the least squares estimate. Several illustrative numerical examples are given to support the theoretical work.
\end{abstract}

Keywords: Bass model, least squares estimate, existence problem, data fitting.

\section{Introduction}

The most popular first-purchase (adoption) diffusion model in marketing research is the Bass model. It is similar in some respect to models of infectious diseases or contagion models which describe the spread of a disease through the population due to contact with infected persons (see Bailey 1975; 1957). The Bass model is distinguished from other growth models by explicitly incorporating some key behavioural assumptions from Rogers' theory of diffusion of innovation (see Rogers, 1962). Namely, Bass divided adopters (first-time buyers) into innovators and imitators. Imitators, unlike innovators, are buyers who are influenced in their adoption by the number of previous buyers. The Bass model has three parameters: the coefficient of innovation or external influence $(p>0)$, the coefficient of imitation or internal influence $(q \geq 0)$, and the total market potential $(m>$ $0)$. To capture the growth of a new durable product (innovation) due to the diffusion effect, Bass (1969) used the following Riccati differential equation with constant coefficients:

$$
\begin{array}{r}
\frac{\mathrm{d} N(t)}{\mathrm{d} t}=p[m-N(t)]+\frac{q}{m} N(t)[m-N(t)], \\
N(0)=0, \quad t \geq 0,
\end{array}
$$

where $N(t)$ and $n(t):=\mathrm{d} N(t) / \mathrm{d} t$ are respectively the cumulative and the noncumulative number of adopters of a new product at time $t$. The adoption rate $n(t)$ is determined by two additive terms: the first term, $p[m-$ $N(t)$, represents adoptions due to innovators, whereas the second term, $(q / m) N(t)[m-N(t)]$, represents adoptions due to imitators.

To stress the fact that functions $N(t)$ and $n(t)$ depend on parameters $m, p$ and $q$, we shall write $N(t ; m, p, q)$ and $n(t ; m, p, q)$.

The solution of (1) and the corresponding adoption rate function are given by

$$
N(t ; m, p, q)=m \frac{1-\mathrm{e}^{-(p+q) t}}{1+\frac{q}{p} \mathrm{e}^{-(p+q) t}}, \quad t \geq 0,
$$

and

$$
n(t ; m, p, q)=m \frac{(p+q)^{2}}{p} \frac{\mathrm{e}^{-(p+q) t}}{\left(1+\frac{q}{p} \mathrm{e}^{-(p+q) t}\right)^{2}}, \quad t \geq 0
$$

The graph of the function $N$ is known as the Bass cumulative adoption curve, and the graph of the function $n$ is known as the Bass (noncumulative) adoption curve.

The graph of the cumulative adoption curve $N$ is an "S-shaped" curve. If $q>p$, for this curve the point of 


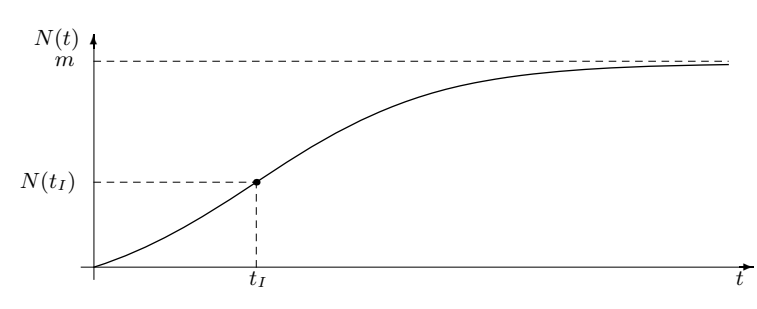

Fig. 1. Typical S-shaped Bass cumulative adoption curve.

inflection occurs at

$$
t_{I}:=\frac{1}{p+q} \ln (q / p)
$$

with

$$
N\left(t_{I} ; m, p, q\right)=m \frac{(q-p)}{2 q}
$$

(see Fig. 1). For $q \leq p$, the graph is still $\mathrm{S}$-shaped, but the point of inflection occurs at a negative value of $t$. Furthermore, if $q>p$, it can be easily shown that the adoption rate function $n$ has a maximum value at $t_{I}$,

$$
n\left(t_{I} ; m, p, q\right)=m \frac{(p+q)^{2}}{4 q},
$$

and that $n$ is symmetric about the peak $t_{I}$. In the case when $q \leq p$, the adoption rate function $n$ is strictly decreasing on $[0, \infty)$ (see Fig. 2).

There are many applications of the Bass model in several areas like retail service, industrial technology, agricultural, educational, pharmaceutical, and consumer durable goods markets. For a review of the Bass model and its applications, see the work of Mahajan et al. (2000).

In practice, the unknown parameters of the Bass model are not known in advance and they must be estimated on the basis of some experimentally or empirically obtained data. This issue is known as a parameter estimation problem. There is no unique way to estimate the unknown parameters and many different methods have been proposed in the literature. Mahajan et al. (1986) used real diffusion data for seven products to compare the performance of four estimation procedures: Ordinary Least Squares (OLS) estimation proposed by

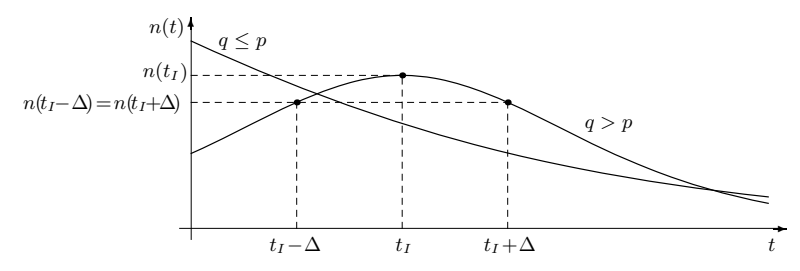

Fig. 2. Symmetry of the Bass adoption curve.
Bass, Maximum Likelihood Estimation (MLE) proposed by Schmittlein and Mahajan (1982), Nonlinear Least Squares (NLS) estimation suggested by Srinivasan and Mason (1986), and Algebraic Estimation (AE) proposed by Mahajan and Sharma (1986). They concluded that, for the seven data sets considered in their study, the NLS procedure provides better predictions as well as more valid estimates of standard errors for the parameter estimates than the other three estimation procedures.

The formulation of the NLS approach is as follows: The observed number of adopters $X_{i}$ in the time interval $\left(\tau_{i-1}, \tau_{i}\right]$ is modeled as

$$
\begin{aligned}
X_{i}=N\left(\tau_{i} ; m, p, q\right)-N\left(\tau_{i-1} ; m, p, q\right) & +\varepsilon_{i}, \\
i & =1, \ldots, K
\end{aligned}
$$

where $\varepsilon_{i}$ is an additive error term. Here, by definition, $\tau_{0}=0$. Based on these equations, Srinivasan and Mason proposed to estimate the unknown parameters $p, q$ and $m$ in the sense of Least Squares (LS) by minimizing functional

$$
\begin{aligned}
& S(m, p, q) \\
& =\sum_{i=1}^{K}\left[X_{i}-\left(N\left(\tau_{i} ; m, p, q\right)-N\left(\tau_{i-1} ; m, p, q\right)\right)\right]^{2}
\end{aligned}
$$

on the set $\{(m, p, q): m, p>0, q \geq 0\}$.

There are several other methods which can be used to estimate the unknown parameters in new product diffusion models (see, e.g., Scitovski and Meler, 2002). A very popular technique for parameter estimation is the least squares method. Numerical methods for solving the nonlinear LS problem are described by Dennis and Schnabel (1996) as well as Gill et al. (1981). Before starting an iterative procedure one should ask whether the LS estimate exists. For nonlinear LS problems this question is difficult to answer. The problem of nonlinear weighted LS and total least squares fitting of the Bass curve (2) is considered by Jukić (2013; 2011). Results on the existence of the LS estimate for some other special classes of functions can be found in the works of Bates and Watts (1988), Björck (1996), Demidenko (2008; 2006; 1996), Hadeler et al. (2007), Jukić (2013; 2009), Jukić and Marković (2010), Jukić et al. (2008; 2004), Marković and Jukić (2010), as well as Marković et al. (2009).

In this paper, we consider the parameter estimation approach for the Bass model, based on nonlinear weighted LS fitting of the Bass adoption curve (3). In Section 2 . we briefly describe this approach and summarize our main results. We show that it is possible that the LS estimate for the Bass adoption curve does not exist (Proposition 1). As our main results, we present two theorems (Theorems 1 and 2) on the existence of the LS estimate, as well as their generalizations (Theorems 3 and 4) in the $l_{s}$ norm $(1 \leq s<\infty)$. Some numerical experiments 
to illustrate the efficiency of our approach are given in Section 3. To compare our approach with the NLS one proposed by Srinivasan and Mason, we used the same time series data for the seven durables. To avoid unnecessary technicalities at an early stage, all proofs are given in Section 2.3. To the best of our knowledge, there is no previous paper that has focused on the existence of the LS estimate for the Bass adoption curve.

\section{Main results: $\mathrm{LS}$ regression existence theorems for the Bass adoption curve}

In this section, we first formulate the LS fitting problem for the Bass adoption curve and then present two theorems (Theorems 1 and 2 on the existence of the least squares estimate, as well as their generalizations (Theorems 3 and (4) in the $l_{s}$ norm $(1 \leq s<\infty)$. Their proofs are given in Section 2.3

2.1. LS fitting problem for the Bass adoption curve. Suppose we are given the data $\left(w_{i}, t_{i}, y_{i}\right), i=1, \ldots, K$, $K>3$, where

$$
0<t_{1}<t_{2}<\ldots<t_{K}
$$

denote the values of the independent variable,

$$
y_{1}, \ldots, y_{K}>0
$$

are in some way obtained respective estimates of the Bass adoption curve (3), i.e., $y_{i} \approx n\left(t_{i} ; m, p, q\right), i=1, \ldots, K$, and $w_{i}>0$ are the data weights which describe the assumed relative accuracy of the data. The unknown parameters $m, p$ and $q$ of the function (3) have to be estimated by minimizing the functional

$$
\begin{aligned}
F & (m, p, q) \\
& =\sum_{i=1}^{K} w_{i}\left[n\left(t_{i} ; m, p, q\right)-y_{i}\right]^{2} \\
& =\sum_{i=1}^{K} w_{i}\left[\frac{m(p+q)^{2}}{p} \frac{\mathrm{e}^{-(p+q) t_{i}}}{\left(1+\frac{q}{p} \mathrm{e}^{-(p+q) t_{i}}\right)^{2}}-y_{i}\right]^{2}
\end{aligned}
$$

on the set

$$
\mathcal{P}:=\{(m, p, q): m, p>0, q \geq 0\} .
$$

A point $\left(m^{\star}, p^{\star}, q^{\star}\right) \in \mathcal{P}$ such that $F\left(m^{\star}, p^{\star}, q^{\star}\right)=$ $\inf _{(m, p, q) \in \mathcal{P}} F(m, p, q)$ is called the least squares estimate, if it exists (see Björck, 1996; Gill et al., 1981; Ross, 1990; Seber and Wild, 1989).

Data for LS estimation can be obtained in various ways. For instance, Eqn. (1) can be discretized in different ways. The most straightforward and most commonly used way is to use the finite difference method, in which case the first derivative is approximated by formulas involving only several neighboring points. To be a bit concrete, let us concentrate only on the three commonly used finite difference approximations, known as forward, backward and centered finite difference approximation. For this purpose, suppose that the observed cumulative number of adopters at times $0<\tau_{1}<\tau_{2}<\ldots<\tau_{K}$ is $N_{1}, N_{2}, \ldots, N_{K}$, respectively. Then the observed number of adopters in the interval $\left(\tau_{i-1}, \tau_{i}\right]$ is given by

$$
X_{i}=N_{i}-N_{i-1}, \quad i=1, \ldots, K,
$$

where $\tau_{0}=0$ and $N_{0}=0$ by definition. The forward, backward and centered finite difference discretizations of differential equation (1) and points $\left(t_{i}, y_{i}\right)$ required for LS estimation are as follows:

- the forward finite difference discretization,

$$
\begin{aligned}
\frac{X_{i}}{\tau_{i}-\tau_{i-1}} & =n\left(\tau_{i-1} ; m, p, q\right)+\varepsilon_{i}, \quad i=1, \ldots, K, \\
\left(t_{i}, y_{i}\right) & =\left(\tau_{i-1}, \frac{X_{i}}{\tau_{i}-\tau_{i-1}}\right), \quad i=1, \ldots, K ;
\end{aligned}
$$

- the backward finite difference discretization

$$
\begin{aligned}
\frac{X_{i}}{\tau_{i}-\tau_{i-1}} & =n\left(\tau_{i} ; m, p, q\right)+\varepsilon_{i}, \quad i=1, \ldots, K, \\
\left(t_{i}, y_{i}\right) & =\left(\tau_{i}, \frac{X_{i}}{\tau_{i}-\tau_{i-1}}\right), \quad i=1, \ldots, K ;
\end{aligned}
$$

- the centered finite difference discretization

$$
\begin{aligned}
\frac{X_{i}}{\tau_{i}-\tau_{i-1}} & =n\left(\frac{\tau_{i-1}+\tau_{i}}{2} ; m, p, q\right)+\varepsilon_{i}, \\
i & =1, \ldots, K \\
\left(t_{i}, y_{i}\right) & =\left(\frac{\tau_{i-1}+\tau_{i}}{2}, \frac{X_{i}}{\tau_{i}-\tau_{i-1}}\right), \\
i & =1, \ldots, K .
\end{aligned}
$$

The following proposition shows that there exist data such that the LS estimate for the Bass adoption curve (3) does not exist.

Proposition 1. Let $\left(w_{i}, t_{i}, y_{i}\right), i=1, \ldots, K, K>3$, be the data such that the points $\left(t_{i}, y_{i}\right), i=1, \ldots, K$, all lie on some exponential curve $y(t)=b \mathrm{e}^{c t}, b, c>0$. Then the LS estimate for the Bass adoption curve (3) does not exist.

Proof. Since $F(m, p, q) \geq 0$ for all $(m, p, q) \in \mathcal{P}$, and

$$
\begin{aligned}
\lim _{x \rightarrow \infty} F( & \left.\frac{b x}{c}, \frac{c}{x+1}, \frac{c x}{x+1}\right) \\
= & \lim _{x \rightarrow \infty} \sum_{i=1}^{K} w_{i}\left[b x \frac{(1+x) \mathrm{e}^{-c t_{i}}}{\left(1+x \mathrm{e}^{-c t_{i}}\right)^{2}}-y_{i}\right]^{2} \\
& =\sum_{i=1}^{K} w_{i}\left(b \mathrm{e}^{c t_{i}}-y_{i}\right)^{2}=0
\end{aligned}
$$


this means that

$$
\inf _{(m, p, q) \in \mathcal{P}} F(m, p, q)=0 .
$$

Furthermore, since the graph of any function of type (3) intersects the graph of exponential function $y(t)=b \mathrm{e}^{c t}$ at no more than three points, and $K>3$, it follows that $F(m, p, q)>0$ for all $(m, p, q) \in \mathcal{P}$, and hence the LS estimate does not exist.

2.2. Existence theorems. The following theorem, whose proof is given in Section 2.3 gives a necessary and sufficient condition on the data which guarantee the existence of the LS estimate for the function (3). First, let us introduce the following notation: Let $E^{\star}$ be an infimum of the weighted sum of squares for the exponential function $y(t)=b \mathrm{e}^{c t}(b, c>0)$, i.e.,

$$
E^{\star}:=\inf _{b, c>0} \sum_{i=1}^{K} w_{i}\left(b \mathrm{e}^{c t_{i}}-y_{i}\right)^{2} .
$$

Theorem 1. Suppose that the data $\left(w_{i}, t_{i}, y_{i}\right), i=$ $1, \ldots, K, K>3$, satisfy the conditions (5) and (6). Then the LS estimate for the Bass adoption curve (3) exists if and only if there is a point $\left(m_{0}, p_{0}, q_{0}\right) \in \mathcal{P}$ such that $F\left(m_{0}, p_{0}, q_{0}\right) \leq E^{\star}$.

In other words, under the assumptions of the theorem, the LS estimate exists if and only if there is at least one regression curve defined by (3) which is in an LS sense as good as 'or better than' the best exponential curve of type $t \mapsto b \mathrm{e}^{c t}$, where $b, c>0$.

It is clear that, regardless of how much effort is put into marketing, there is a certain upper bound, say $M$, for the market potential $m$ (i.e., the maximum number of adopters). In most cases management has a judgement, a strong intuitive feel, about the upper bound $M$, but if not, the upper bound $M$ can be the size of the relevant population. The following theorem tells us that if parameter $m$ is bounded above, then the LS estimate will exist. First, let us introduce the following notation: Given any real number $M>0$, let

$$
\mathcal{P}_{M}:=\{(m, p, q): 0<m \leq M, p>0, q \geq 0\} .
$$

Theorem 2. Suppose that the data $\left(w_{i}, t_{i}, y_{i}\right), i=$ $1, \ldots, K, K>3$, satisfy the conditions (5) and (6). Then functional $F$ defined by (7) attains its infimum on $\mathcal{P}_{M}$, i.e., there exists a point $\left(m^{\star}, p^{\star}, q^{\star}\right) \in \mathcal{P}_{M}$ such that $F\left(m^{\star}, p^{\star}, q^{\star}\right)=\inf _{(m, p, q) \in \mathcal{P}_{M}} F(m, p, q)$.

The proof of this theorem is the same for respective parts of the proof of Theorem 1 with the exception that we do not have to prove that $m^{\star}<\infty$. Hence, it is omitted.

The LS problem is a nonlinear $l_{2}$-norm one. During the last few decades an increased interest in alternative $l_{s}$-norm has become apparent (see, e.g., Atieg and Watson, 2004; Gonin and Money, 1989). For example, $l_{1}$-norm criteria are more suitable if there are wild points (outliers) in the data. Thus, instead of minimizing functional $F$, sometimes a more adequate criterion for estimation of unknown parameters $m, p$ and $q$ of the function (3) is to minimize the following functional:

$$
F_{s}(m, p, q)=\sum_{i=1}^{K} w_{i}\left|n\left(t_{i} ; m, p, q\right)-y_{i}\right|^{s}
$$

where $s(1 \leq s<\infty)$ is an arbitrary fixed number. To state the corresponding $l_{s}$-norm $(1 \leq s<\infty)$ generalizations of Theorems 1 and 2, we need an additional notation. Let

$$
E_{s}^{\star}:=\inf _{(b, c) \in \mathbb{R}_{+}^{2}} E_{s}(b, c),
$$

where

$$
E_{s}(b, c)=\sum_{i=1}^{K} w_{i}\left|b \mathrm{e}^{c t_{i}}-y_{i}\right|^{s} .
$$

Obviously, $E^{\star}=E_{2}^{\star}$ and $F=F_{2}$.

Theorem 3. If the data $\left(w_{i}, t_{i}, y_{i}\right), i=1, \ldots, K, K>$ 3, satisfy the conditions (5) and (6), then functional $F_{s}$ defined by (8) attains its infimum on $\mathcal{P}$ if and only if there is a point $\left(m_{0}, p_{0}, q_{0}\right) \in \mathcal{P}$ such that $F_{s}\left(m_{0}, p_{0}, q_{0}\right) \leq$ $E_{s}^{\star}$.

The proof of the following theorem is also omitted; it is the same for the respective parts of the proof of Theorem 3, with the exception that we do not have to prove that $m^{\star}<\infty$.

Theorem 4. If the data $\left(w_{i}, t_{i}, y_{i}\right), i=1, \ldots, K, K>3$, satisfy the conditions (5) and (6), then there exists a point $\left(m^{\star}, p^{\star}, q^{\star}\right) \in \mathcal{P}_{M}$ such that

$$
F_{s}\left(m^{\star}, p^{\star}, q^{\star}\right)=\inf _{(m, p, q) \in \mathcal{P}_{M}} F_{s}(m, p, q) .
$$

2.3. Proofs of Theorems 1 and 3. The following lemma will be used in proofs of both Theorems 1 and 3 .

Lemma 1. Suppose that the data $\left(w_{i}, t_{i}, y_{i}\right), i=$ $1, \ldots, K, K>3$, satisfy the conditions (5) and (6). Then given any $i_{0} \in\{1, \ldots, K-1\}$ there exists a point in $\mathcal{P}$ at which functional $F_{s}$ defined by (8) attains a value less than

$$
\sum_{\substack{i=1 \\ i \neq i_{0}, i_{0}+1}}^{K} w_{i}\left|y_{i}\right|^{s}
$$


Proof. Let us first write

$$
x_{0}:=\frac{1}{t_{i_{0}+1}-t_{i_{0}}} \max \left\{\ln \left(\frac{y_{i_{0}+1}}{y_{i_{0}}}\right), \ln \left(\frac{y_{i_{0}}}{y_{i_{0}+1}}\right)\right\},
$$

and then define functions $\alpha, m, p, q:\left(x_{0}, \infty\right) \rightarrow(0, \infty)$ as follows:

$$
\begin{aligned}
\alpha(x) & :=\frac{1-\sqrt{\frac{y_{i_{0}}}{y_{i_{0}}+1}} \mathrm{e}^{-\frac{x}{2}\left(t_{i_{0}+1}-t_{i_{0}}\right)}}{\sqrt{\frac{y_{i_{0}}}{y_{i_{0}}+1}}-\mathrm{e}^{-\frac{x}{2}\left(t_{i_{0}+1}-t_{i_{0}}\right)}}, \\
m(x) & :=\frac{y_{i_{0}+1}\left[1+\alpha(x) \mathrm{e}^{-\frac{x}{2}\left(t_{i_{0}+1}-t_{i_{0}}\right)}\right]^{2} \mathrm{e}^{x t_{i_{0}+1}}}{x\left[1+\alpha(x) \mathrm{e}^{\frac{x}{2}\left(t_{i_{0}}+t_{i_{0}}+1\right)}\right]} \\
p(x) & :=\frac{x}{1+\alpha(x) \mathrm{e}^{\frac{x}{2}\left(t_{i_{0}}+t_{i_{0}}+1\right)}}, \\
q(x) & :=\frac{x \alpha(x) \mathrm{e}^{\frac{x}{2}\left(t_{i_{0}}+t_{i_{0}+1}\right)}}{1+\alpha(x) \mathrm{e}^{\frac{x}{2}\left(t_{i_{0}}+t_{i_{0}+1}\right)}} .
\end{aligned}
$$

By using the definition of $x_{0}$, it is easy to show that function $\alpha$ is well defined and strictly positive on $\left(x_{0}, \infty\right)$. Thus for all $x \in\left(x_{0}, \infty\right)$ we have that $(m(x), p(x), q(x)) \in \mathcal{P}$. Furthermore, it is easy to verify that

$$
\begin{aligned}
& n(t ; m(x), p(x), q(x)) \\
& =y_{i_{0}+1}\left[1+\alpha(x) \mathrm{e}^{-\frac{x}{2}\left(t_{i_{0}+1}-t_{i_{0}}\right)}\right]^{2} \\
& \quad \times \frac{\mathrm{e}^{-x\left(t-t_{i_{0}+1}\right)}}{\left[1+\alpha(x) \mathrm{e}^{-x\left(t-\frac{t_{i_{0}}+t_{i_{0}}+1}{2}\right)}\right]^{2}} .
\end{aligned}
$$

Now, by a straightforward but tedious calculation, one can verify that, for all $x \in\left(x_{0}, \infty\right)$,

$$
\begin{gathered}
n\left(t_{i_{0}} ; m(x), p(x), q(x)\right)=y_{i_{0}}, \\
n\left(t_{i_{0}+1} ; m(x), p(x), q(x)\right)=y_{i_{0}+1},
\end{gathered}
$$

and

$$
\begin{aligned}
\lim _{x \rightarrow \infty} n(t ; m(x), p(x), q(x)) \\
\quad=\left\{\begin{aligned}
0 & \text { if } t \in\left(-\infty, t_{i_{0}}\right) \cup\left(t_{i_{0}+1}, \infty\right), \\
\infty & \text { if } t \in\left(t_{i_{0}}, t_{i_{0}+1}\right) .
\end{aligned}\right.
\end{aligned}
$$

In Fig. 3 we plot the graph of the function $t \mapsto$ $n(t ; m(x), p(x), q(x))$.

Let $x>x_{0}$ be sufficiently large, so that

$$
0<n\left(t_{i} ; m(x), p(x), q(x)\right) \leq y_{i}, \quad i=1, \ldots, K,
$$

whereby the equality holds only if $i=i_{0}$ or $i=i_{0}+1$. Due to the above mentioned facts, such $x$ exists. Then

$$
\begin{aligned}
& F_{s}(m(x), p(x), q(x)) \\
& =\sum_{i=1}^{K} w_{i}\left|n\left(t_{i} ; m(x), p(x), q(x)\right)-y_{i}\right|^{s} \\
& <\sum_{\substack{i=1 \\
i \neq i_{0}, i_{0}+1}}^{K} w_{i}\left|y_{i}\right|^{s} .
\end{aligned}
$$

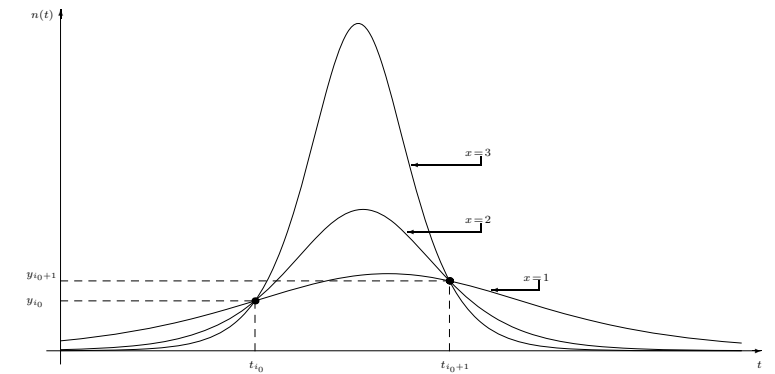

Fig. 3. Plots of the Bass adoption curve $n(t ; m(x), p(x), q(x))$ for some values of $x$.

Proof. (Theorem 1) Assume first that $\left(m^{\star}, p^{\star}, q^{\star}\right) \in \mathcal{P}$ is the LS estimate, and then show that $F\left(m^{\star}, p^{\star}, q^{\star}\right) \leq E^{\star}$. In order to do this, first note that, for all $b, c, x>0$,

$$
\begin{aligned}
F\left(m^{\star}, p^{\star}, q^{\star}\right) & \leq F\left(\frac{b x}{c}, \frac{c}{x+1}, \frac{c x}{x+1}\right) \\
& =\sum_{i=1}^{K} w_{i}\left[x b \frac{(1+x) \mathrm{e}^{-c t_{i}}}{\left(1+x \mathrm{e}^{-c t_{i}}\right)^{2}}-y_{i}\right]^{2},
\end{aligned}
$$

from where taking the limit as $x \rightarrow \infty$ it follows that (see the proof of Proposition 1

$$
F\left(m^{\star}, p^{\star}, q^{\star}\right) \leq \sum_{i=1}^{K} w_{i}\left(b \mathrm{e}^{c t_{i}}-y_{i}\right)^{2} .
$$

From the last inequality and the definition of $E^{\star}$ we obtain that $F\left(m^{\star}, p^{\star}, q^{\star}\right) \leq E^{\star}$.

Let us show the converse of the theorem. Suppose that there is a point $\left(m_{0}, p_{0}, q_{0}\right) \in \mathcal{P}$ such that $F\left(m_{0}, p_{0}, q_{0}\right) \leq E^{\star}$. Since the functional $F$ is nonnegative, there exists $F^{\star}:=\inf _{(m, p, q) \in \mathcal{P}} F(m, p, q)$. It should be shown that the LS estimate exists, i.e., that there exists a point $\left(m^{\star}, p^{\star}, q^{\star}\right) \in \mathcal{P}$ such that $F\left(m^{\star}, p^{\star}, q^{\star}\right)=F^{\star}$. To do this, first note that

$$
F^{\star} \leq F\left(m_{0}, p_{0}, q_{0}\right) \leq E^{\star} .
$$

If $F^{\star}=F\left(m_{0}, p_{0}, q_{0}\right)$, to complete the proof it is enough to set $\left(m^{\star}, p^{\star}, q^{\star}\right)=\left(m_{0}, p_{0}, q_{0}\right)$. Hence, we can further assume that

$$
F^{\star}<F\left(m_{0}, p_{0}, q_{0}\right) \leq E^{\star} .
$$

Let $\left(m_{k}, p_{k}, q_{k}\right)$ be a sequence in $\mathcal{P}$, such that

$$
\begin{aligned}
F^{\star} & =\lim _{k \rightarrow \infty} F\left(m_{k}, p_{k}, q_{k}\right) \\
& =\lim _{k \rightarrow \infty} \sum_{i=1}^{K} w_{i}\left[n\left(t_{i} ; m_{k}, p_{k}, q_{k}\right)-y_{i}\right]^{2} .
\end{aligned}
$$

Without loss of generality, in further deliberations we may assume that sequences $\left(m_{k}\right),\left(p_{k}\right)$ and $\left(q_{k}\right)$ are monotone. 
This is possible because the sequence $\left(m_{k}, p_{k}, q_{k}\right)$ has a subsequence $\left(m_{l_{k}}, p_{l_{k}}, q_{l_{k}}\right)$, such that all its component sequences $\left(m_{l_{k}}\right),\left(p_{l_{k}}\right)$ and $\left(q_{l_{k}}\right)$ are monotone, and since $\lim _{k \rightarrow \infty} F\left(m_{l_{k}}, p_{l_{k}}, q_{l_{k}}\right)=\lim _{k \rightarrow \infty} F\left(m_{k}, p_{k}, q_{k}\right)=$ $F^{\star}$.

Since each monotone sequence of real numbers converges in the extended real number system $\overline{\mathbb{R}}$, define

$$
m^{\star}:=\lim _{k \rightarrow \infty} m_{k}, \quad p^{\star}:=\lim _{k \rightarrow \infty} p_{k}, \quad q^{\star}:=\lim _{k \rightarrow \infty} q_{k} .
$$

Note that $0 \leq m^{\star}, p^{\star}, q^{\star} \leq \infty$, because $\left(m_{k}, p_{k}, q_{k}\right) \in$ $\mathcal{P}$.

To complete the proof, it is enough to show that $\left(m^{\star}, p^{\star}, q^{\star}\right) \in \mathcal{P}$, i.e., that $0<m^{\star}<\infty, 0<p^{\star}<\infty$ and $0 \leq q^{\star}<\infty$. The continuity of the functional $F$ will then imply that $F^{\star}=\lim _{k \rightarrow \infty} F\left(m_{k}, p_{k}, q_{k}\right)=$ $F\left(m^{\star}, p^{\star}, q^{\star}\right)$.

It remains to show that $\left(m^{\star}, p^{\star}, q^{\star}\right) \in \mathcal{P}$. The proof will be derived in three steps. In Step 1, we will show that $0<m^{\star}<\infty$. After that, in Step 2, we will show that $0<p^{\star}+q^{\star}<\infty$. The proof that $p^{\star}>0$ will be given in Step 3 .

Step 1. Let us first show that $0<m^{\star}<\infty$. We prove this by contradiction. Suppose on the contrary that $m^{\star}=0$ or $m^{\star}=\infty$. Then only one of the following three cases can occur: (i) $p^{\star}+q^{\star}=0$, (ii) $0<p^{\star}+q^{\star}<\infty$, or (iii) $p^{\star}+q^{\star}=\infty$. Now, we are going to show that the functional $F$ cannot attain its infimum in either of these three cases, which will prove that $0<m^{\star}<\infty$.

Case (i): $p^{\star}+q^{\star}=0$. Let $L:=\lim _{k \rightarrow \infty} m_{k} p_{k}$. First note that $0 \leq L \leq \infty$.

By using the inequality

$$
1<\frac{1+\frac{q_{k}}{p_{k}}}{1+\frac{q_{k}}{p_{k}} \mathrm{e}^{-\left(p_{k}+q_{k}\right) t}}<\mathrm{e}^{\left(p_{k}+q_{k}\right) t} \text { for all } t \geq 0,
$$

it follows readily that

$$
\lim _{k \rightarrow \infty} \frac{1+\frac{q_{k}}{p_{k}}}{1+\frac{q_{k}}{p_{k}} \mathrm{e}^{-\left(p_{k}+q_{k}\right) t}}=1 \text { for all } t \geq 0 .
$$

Hence,

$$
\begin{aligned}
& \lim _{k \rightarrow \infty} n\left(t_{i} ; m_{k}, p_{k}, q_{k}\right) \\
& =\lim _{k \rightarrow \infty}\left[m_{k} p_{k}\left(\frac{1+\frac{q_{k}}{p_{k}}}{1+\frac{q_{k}}{p_{k}} \mathrm{e}^{-\left(p_{k}+q_{k}\right) t_{i}}}\right)^{2} \mathrm{e}^{-\left(p_{k}+q_{k}\right) t_{i}}\right] \\
& =L,
\end{aligned}
$$

$i=1, \ldots, K$. If $L=\infty$, then it would follow that $F^{\star}=$ $\infty$, which is impossible. If $L<\infty$, then we would obtain that

$$
F^{\star}=\sum_{i=1}^{K} w_{i}\left(L-y_{i}\right)^{2} .
$$

Since, by the definition of $E^{\star}$,

$$
\sum_{i=1}^{K} w_{i}\left(L \mathrm{e}^{c t_{i}}-y_{i}\right)^{2} \geq E^{\star} \quad \text { for all } c>0,
$$

taking the limit as $c \rightarrow 0+$ it follows that $\sum_{i=1}^{K} w_{i}(L-$ $\left.y_{i}\right)^{2} \geq E^{\star}$. From this and (11), we would have that $F^{\star} \geq$ $E^{\star}$, which contradicts the assumption (9). This means that in this case the functional $F$ cannot attain its infimum.

Case (ii): $0<p^{\star}+q^{\star}<\infty$. Note that

$$
\begin{aligned}
& n\left(t_{i} ; m_{k}, p_{k}, q_{k}\right) \\
& \quad=m_{k} p_{k}\left(\frac{1+\frac{q_{k}}{p_{k}}}{1+\frac{q_{k}}{p_{k}} \mathrm{e}^{-\left(p_{k}+q_{k}\right) t_{i}}}\right)^{2} \mathrm{e}^{-\left(p_{k}+q_{k}\right) t_{i}},
\end{aligned}
$$

$i=1, \ldots, K$. It readily follows, regardless of whether $q_{k} / p_{k}$ converges to a finite number or diverges to infinity, that there exist all limits

$$
\alpha_{i}^{\star}:=\lim _{k \rightarrow \infty}\left(\frac{1+\frac{q_{k}}{p_{k}}}{1+\frac{q_{k}}{p_{k}} \mathrm{e}^{-\left(p_{k}+q_{k}\right) t_{i}}}\right)^{2}, \quad i=1, \ldots, K,
$$

and that $0<\alpha_{i}^{\star}<\infty$.

Let us first show that $m^{\star} \neq 0$. We prove this by contradiction. Suppose on the contrary that $m^{\star}=0$. Then, by using (12) and (13), it is easy to show that $\lim _{k \rightarrow \infty} n\left(t_{i} ; m_{k}, p_{k}, q_{k}\right)=0$ for all $i=1, \ldots, K$, and therefore it would follow that $F^{\star}=\sum_{i=1}^{K} w_{i} y_{i}^{2}$. Since, according to Lemma1 there exists a point in $\mathcal{P}$ at which the functional $F$ attains a value smaller than $\sum_{i \neq i_{0}} w_{i} y_{i}^{2}$, this means that in this way $\left(m^{\star}=0\right)$ the functional $F$ cannot attain its infimum.

It remains to show that $m^{\star} \neq \infty$. The proof will be given also by contradiction. Assume that $m^{\star}=\infty$. Then by using (12) and (13) one can easy to show that there must be $\lim _{k \rightarrow \infty} p_{k}=p^{\star}=0$, because otherwise we would have that $\lim _{k \rightarrow \infty} n\left(t_{i} ; m_{k}, p_{k}, q_{k}\right)=\infty$ for all $i=1, \ldots, K$, and consequently, $F^{\star}=\infty$, which is, as we know, impossible. Therefore, because $p^{\star}=0$ and $0<p^{\star}+q^{\star}<\infty$, there must be $q^{\star}>0$, which would imply $\lim _{k \rightarrow \infty} q_{k} / p_{k}=\infty$ and, consequently,

$$
\alpha_{i}^{\star}=\mathrm{e}^{2 q^{\star} t_{i}}, \quad i=1, \ldots, K .
$$

Now, by using (12) it is easy to show that

$$
\lim _{k \rightarrow \infty} n\left(t_{i} ; m_{k}, p_{k}, q_{k}\right)=L \mathrm{e}^{q^{\star} t_{i}}, \quad i=1, \ldots, K,
$$

where, as in Case (i), $L=\lim _{k \rightarrow \infty} m_{k} p_{k}$. If $L=\infty$, then it would follow that $F^{\star}=\infty$, which is impossible. If $L<\infty$, then we would obtain that

$$
F^{\star}=\lim _{k \rightarrow \infty} F\left(m_{k}, p_{k}, q_{k}\right)=\sum_{i=1}^{K} w_{i}\left(L \mathrm{e}^{q^{\star} t_{i}}-y_{i}\right)^{2} \geq E^{\star},
$$


which contradicts the assumption (9). This proves that $m^{\star} \neq \infty$.

Case (iii): $p^{\star}+q^{\star}=\infty$. Note that

$$
\begin{gathered}
n\left(t_{i} ; m_{k}, p_{k}, q_{k}\right) \\
=\frac{m_{k}\left(p_{k}+q_{k}\right)}{1+\frac{q_{k}}{p_{k}} \mathrm{e}^{-\left(p_{k}+q_{k}\right) t_{i}}} \frac{\left(1+\frac{q_{k}}{p_{k}}\right) \mathrm{e}^{-\left(p_{k}+q_{k}\right) t_{i}}}{1+\frac{q_{k}}{p_{k}} \mathrm{e}^{-\left(p_{k}+q_{k}\right) t_{i}}}, \\
i=1, \ldots, K .
\end{gathered}
$$

Before continuing the proof, let us also note that

$$
\begin{aligned}
\frac{q_{k}}{p_{k}} \mathrm{e}^{-\left(p_{k}+q_{k}\right) t_{1}} & \geq \frac{q_{k}}{p_{k}} \mathrm{e}^{-\left(p_{k}+q_{k}\right) t_{2}} \\
& \geq \cdots \geq \frac{q_{k}}{p_{k}} \mathrm{e}^{-\left(p_{k}+q_{k}\right) t_{K}} .
\end{aligned}
$$

Now, let us first show that $\lim _{k \rightarrow \infty} q_{k} / p_{k} \mathrm{e}^{-\left(p_{k}+q_{k}\right) t_{i}}$ is either 0 or $\infty$, for all $i=1, \ldots, K$. Assume on the contrary that there exists an index $i_{0}$ such that

$$
0<\lim _{k \rightarrow \infty} \frac{q_{k}}{p_{k}} \mathrm{e}^{-\left(p_{k}+q_{k}\right) t_{i_{0}}}:=L_{i_{0}}<\infty .
$$

Then

$$
\lim _{k \rightarrow \infty} \frac{\left(1+\frac{q_{k}}{p_{k}}\right) \mathrm{e}^{-\left(p_{k}+q_{k}\right) t_{i_{0}}}}{1+\frac{q_{k}}{p_{k}} \mathrm{e}^{-\left(p_{k}+q_{k}\right) t_{i_{0}}}}=\frac{L_{i_{0}}}{1+L_{i_{0}}}>0,
$$

and therefore from (14) it would follow that

$$
\begin{aligned}
\lim _{k \rightarrow \infty} n\left(t_{i_{0}} ; m_{k}, p_{k}, q_{k}\right) & \\
& =\lim _{k \rightarrow \infty} m_{k}\left(p_{k}+q_{k}\right) \frac{L_{i_{0}}}{\left(1+L_{i_{0}}\right)^{2}} .
\end{aligned}
$$

In order to have a bounded functional, the limit $\lim _{k \rightarrow \infty} m_{k}\left(p_{k}+q_{k}\right)$ must be finite (regardless of the value of $\left.m^{\star}\right)$. Due to this and the equality

$$
\frac{q_{k}}{p_{k}} \mathrm{e}^{-\left(p_{k}+q_{k}\right) t_{i}}=\frac{q_{k}}{p_{k}} \mathrm{e}^{-\left(p_{k}+q_{k}\right) t_{i_{0}}} \mathrm{e}^{-\left(p_{k}+q_{k}\right)\left(t_{i}-t_{i_{0}}\right)},
$$

it follows readily that

$$
\lim _{k \rightarrow \infty} \frac{q_{k}}{p_{k}} \mathrm{e}^{-\left(p_{k}+q_{k}\right) t_{i}}= \begin{cases}\infty, & i<i_{0} \\ 0, & i>i_{0}\end{cases}
$$

Now, by using (14) it is easy to check that $\lim _{k \rightarrow \infty} n\left(t_{i} ; m_{k}, p_{k}, q_{k}\right)=0$ for each $i \neq i_{0}$. That would imply that $F^{\star} \geq \sum_{i \neq i_{0}} w_{i} y_{i}^{2}$. But since according to Lemma 1 there exists a point in $\mathcal{P}$ at which the functional $F$ attains a value smaller than $\sum_{i \neq i_{0}} w_{i} y_{i}^{2}$, we have proved that $\lim _{k \rightarrow \infty}\left(q_{k} / p_{k}\right) \mathrm{e}^{-\left(p_{k}+q_{k}\right) t_{i}}$ is either 0 or $\infty$, for all $i=1, \ldots, K$.

Note that only one of the following three subcases can occur: (a) $\lim _{k \rightarrow \infty} \frac{q_{k}}{p_{k}} \mathrm{e}^{-\left(p_{k}+q_{k}\right) t_{i}}=\infty$ for all $i=1, \ldots, K$, (b) $\lim _{k \rightarrow \infty} \frac{q_{k}}{p_{k}} \mathrm{e}^{-\left(p_{k}+q_{k}\right) t_{i}}=0$ for all $i=1, \ldots, K$, or (c) there exists $i_{0} \neq K$ such that

$$
\lim _{k \rightarrow \infty} \frac{q_{k}}{p_{k}} \mathrm{e}^{-\left(p_{k}+q_{k}\right) t_{i}}= \begin{cases}\infty, & i \leq i_{0} \\ 0, & i>i_{0} .\end{cases}
$$

Subcase (a): If $\lim _{k \rightarrow \infty} \frac{q_{k}}{p_{k}} \mathrm{e}^{-\left(p_{k}+q_{k}\right) t_{i}}=\infty$ for all $i=$ $1, \ldots, K$, then

$$
\lim _{k \rightarrow \infty} \frac{\left(1+\frac{q_{k}}{p_{k}}\right) \mathrm{e}^{-\left(p_{k}+q_{k}\right) t_{i}}}{1+\frac{q_{k}}{p_{k}} \mathrm{e}^{-\left(p_{k}+q_{k}\right) t_{i}}}=1, \quad i=1, \ldots, K
$$

and therefore

$$
\begin{aligned}
\lim _{k \rightarrow \infty} & n\left(t_{i} ; m_{k}, p_{k}, q_{k}\right) \\
= & \lim _{k \rightarrow \infty} m_{k} p_{k} \mathrm{e}^{\left(p_{k}+q_{k}\right) t_{i}}\left[\frac{\left(1+\frac{q_{k}}{p_{k}}\right) \mathrm{e}^{-\left(p_{k}+q_{k}\right) t_{i}}}{1+\frac{q_{k}}{p_{k}} \mathrm{e}^{-\left(p_{k}+q_{k}\right) t_{i}}}\right]^{2} \\
= & \lim _{k \rightarrow \infty} m_{k} p_{k} \mathrm{e}^{\left(p_{k}+q_{k}\right) t_{i}}, \quad i=1, \ldots, K .
\end{aligned}
$$

To keep the functional $F$ bounded, the limit $\lim _{k \rightarrow \infty} m_{k} p_{k} \mathrm{e}^{\left(p_{k}+q_{k}\right) t_{K}}$ must be finite. Because of that, by using the equality

$$
m_{k} p_{k} \mathrm{e}^{\left(p_{k}+q_{k}\right) t_{i}}=m_{k} p_{k} \mathrm{e}^{\left(p_{k}+q_{k}\right) t_{K}} \mathrm{e}^{\left(p_{k}+q_{k}\right)\left(t_{i}-t_{K}\right)}
$$

it follows directly that

$$
\lim _{k \rightarrow \infty} m_{k} p_{k} \mathrm{e}^{\left(p_{k}+q_{k}\right) t_{i}}=0,
$$

i.e.,

$$
\lim _{k \rightarrow \infty} n\left(t_{i} ; m_{k}, p_{k}, q_{k}\right)=0
$$

for all $i=1, \ldots, K-1$. In this subcase we would have

$$
F^{\star}=\lim _{k \rightarrow \infty} F\left(m_{k}, p_{k}, q_{k}\right) \geq \sum_{i=1}^{K-1} w_{i} y_{i}^{2} .
$$

Subcase (b): Let us assume that

$$
\lim _{k \rightarrow \infty} \frac{q_{k}}{p_{k}} \mathrm{e}^{-\left(p_{k}+q_{k}\right) t_{i}}=0
$$

for all $i=1, \ldots, K$. Then

$$
\lim _{k \rightarrow \infty} \frac{\frac{p_{k}}{q_{k}} \mathrm{e}^{\left(p_{k}+q_{k}\right) t_{i}}}{1+\frac{p_{k}}{q_{k}} \mathrm{e}^{\left(p_{k}+q_{k}\right) t_{i}}}=1, \quad i=1, \ldots, K
$$

and thus

$$
\begin{aligned}
& \lim _{k \rightarrow \infty} n\left(t_{i} ; m_{k}, p_{k}, q_{k}\right) \\
& =\lim _{k \rightarrow \infty} m_{k} \frac{\left(p_{k}+q_{k}\right)^{2}}{p_{k}} \mathrm{e}^{-\left(p_{k}+q_{k}\right) t_{i}}\left[\frac{\frac{p_{k}}{q_{k}} \mathrm{e}^{\left(p_{k}+q_{k}\right) t_{i}}}{1+\frac{p_{k}}{q_{k}} \mathrm{e}^{\left(p_{k}+q_{k}\right) t_{i}}}\right]^{2} \\
& =\lim _{k \rightarrow \infty} m_{k} \frac{\left(p_{k}+q_{k}\right)^{2}}{p_{k}} \mathrm{e}^{-\left(p_{k}+q_{k}\right) t_{i}}, \quad i=1, \ldots, K .
\end{aligned}
$$


Proceeding similarly as in the previous subcase, it can be shown that

$$
\lim _{k \rightarrow \infty} n\left(t_{i} ; m_{k}, p_{k}, q_{k}\right)=0, \quad i=2, \ldots, K,
$$

and therefore in this subcase we would have

$$
F^{\star}=\lim _{k \rightarrow \infty} F\left(m_{k}, p_{k}, q_{k}\right) \geq \sum_{i=2}^{K} w_{i} y_{i}^{2} .
$$

Subcase (c): Arguing similarly as in subcases (a) and (b), it can be shown that

$$
\begin{aligned}
\lim _{k \rightarrow \infty} n\left(t_{i} ; m_{k}, p_{k}, q_{k}\right) & =0, \\
i & \in\{1, \ldots, K\} \backslash\left\{i_{0}, i_{0}+1\right\},
\end{aligned}
$$

and therefore in this subcase we would have

$$
F^{\star}=\lim _{k \rightarrow \infty} F\left(m_{k}, p_{k}, q_{k}\right) \geq \sum_{\substack{i=1 \\ i \neq i_{0}, i_{0}+1}}^{K} w_{i} y_{i}^{2} .
$$

Since according to Lemma 11 in each of Subcases (a)-(c) there exists a point in $\mathcal{P}$ at which the functional $F$ attains a value smaller than $\lim _{k \rightarrow \infty} F\left(m_{k}, p_{k}, q_{k}\right)$, our functional $F$ cannot attain its infimum in either of these three subcases, regardless of whether $m^{\star}=0$ or $m^{\star}=\infty$.

So far we have shown that $0<m^{\star}<\infty$, and this will be used in the sequel.

Step 2. Let us first show that $p^{\star}+q^{\star}>0$. We prove this by contradiction. Suppose on the contrary that $p^{\star}+q^{\star}=$ 0 . Then from the inequalities

$$
1<\frac{1+\frac{q_{k}}{p_{k}}}{1+\frac{q_{k}}{p_{k}} \mathrm{e}^{-\left(p_{k}+q_{k}\right) t_{i}}}<\mathrm{e}^{\left(p_{k}+q_{k}\right) t_{i}}, \quad i=1, \ldots, K
$$

it follows that

$$
\lim _{k \rightarrow \infty} \frac{1+\frac{q_{k}}{p_{k}}}{1+\frac{q_{k}}{p_{k}} \mathrm{e}^{-\left(p_{k}+q_{k}\right) t_{i}}}=1, \quad i=1, \ldots, K .
$$

Accordingly, now it is easy to show that

$$
\begin{aligned}
& \lim _{k \rightarrow \infty} n\left(t_{i} ; m_{k}, p_{k}, q_{k}\right) \\
& \quad=\lim _{k \rightarrow \infty} m_{k} p_{k}\left[\frac{1+\frac{q_{k}}{p_{k}}}{1+\frac{q_{k}}{p_{k}} \mathrm{e}^{-\left(p_{k}+q_{k}\right) t_{i}}}\right]^{2} \mathrm{e}^{-\left(p_{k}+q_{k}\right) t_{i}}=0,
\end{aligned}
$$

$i=1, \ldots, K$, and therefore from (10) it would follow that $F^{\star}=\sum_{i=1}^{K} w_{i} y_{i}^{2}$. Since according to Lemma 1 there exists a point in $\mathcal{P}$ at which functional $F$ attains a value smaller than $\sum_{i=1}^{K} w_{i} y_{i}^{2}$, this means that in this way functional $F$ cannot attain its infimum. Thus, we have proved that $p^{\star}+q^{\star}>0$.
The proof that $p^{\star}+q^{\star}<\infty$ can be given by contradiction. To do this, it is sufficient to proceed as in Case (iii) from Step 1.

In this way we have completed the proof that $0<$ $p^{\star}+q^{\star}<\infty$.

Step 3. It remains to show that $p^{\star}>0$. Suppose on the contrary that $p^{\star}=0$. Then from the inequalities $0<$ $p^{\star}+q^{\star}<\infty$ it follows that $q^{\star}>0$, and consequently $\lim _{k \rightarrow \infty} \frac{q_{k}}{p_{k}}=\infty$. Now it is easy to conclude that

$$
\begin{aligned}
\lim _{k \rightarrow \infty} n\left(t_{i} ; m_{k}, p_{k}, q_{k}\right) \\
\quad=\lim _{k \rightarrow \infty} m_{k} p_{k}\left[\frac{1+\frac{q_{k}}{p_{k}}}{1+\frac{q_{k}}{p_{k}} \mathrm{e}^{-\left(p_{k}+q_{k}\right) t_{i}}}\right]^{2} \mathrm{e}^{-\left(p_{k}+q_{k}\right) t_{i}}=0,
\end{aligned}
$$

$i=1, \ldots, K$. As shown in Step 2, in this way functional $F$ cannot attain its infimum. Thus, we proved that $p^{\star}>0$ and herewith we have completed the proof.

Proof. (Theorem 3) The proof of Theorem 3 is similar to that of Theorem 11 just replace the $l_{2}$ norm with the $l_{s}$ norm. Thereby all parts of the proof remain the same.

\section{Numerical experiments}

In the following examples, the obtained estimates of the optimal parameters $\left(m^{\star}, p^{\star}, q^{\star}\right)$ will be denoted by $\left(\bar{m}^{\star}, \bar{p}^{\star}, \bar{q}^{\star}\right)$.

Example 1. To illustrate the accuracy of the parameter estimate approach for the Bass model based on the LS fitting adoption curve, we start with the following data which satisfy the exact solution $(m=1000, p=$ $0.001, q=0.2$ ) of the differential equation (11):

$$
\tau_{i}=i, \quad N_{i}=N\left(\tau_{i} ; 1000,0.001,0.2\right),
$$

$i=1, \ldots, 53$. These data have a point of inflection where

$$
t_{I}=\frac{1}{p+q} \ln (q / p)=26.3598 .
$$

We analyzed three sets of data: the data up to the point just before the point of inflection $(K=26)$, the data up to the point just after the point of inflection $(K=27)$, and the data up to the ceiling ( $K=53)$. The results of the LS fitting Bass adoption curve to corresponding data obtained by using the centered finite approximation are given in Table 1 For all weights $w_{i}$ we took 1.

Example 2. Let $\left(\tau_{i}, N_{i}\right), i=1, \ldots, K$, be the data where

$$
\begin{aligned}
& K=50, \quad \tau_{i}=i, \quad i=1, \ldots, K, \\
& N_{i}=N\left(\tau_{i} ; 1000,0.001,0.2\right)+\varepsilon_{i}, \quad \varepsilon_{i}=\mathcal{N}\left(0, \sigma^{2}\right) .
\end{aligned}
$$

As measures of the quality of fitting, we will use the Mean Absolute Relative Error (MARE) and the Root 
Table 1. Accuracy of parameter estimates.

\begin{tabular}{|c|c|c|c|c|c|c|}
\hline & $\bar{m}^{\star}$ & $\left|\frac{\bar{m}^{\star}-m}{m}\right| \cdot 100$ & $\bar{p}^{\star}$ & $\left|\frac{\bar{p}^{\star}-p}{p}\right| \cdot 100$ & $\bar{q}^{\star}$ & $\left|\frac{\bar{q}^{\star}-q}{q}\right| \cdot 100$ \\
\hline \hline$K=26$ & 999.486 & 0.0514 & 0.00100308 & 0.3080 & 0.199901 & 0.0495 \\
$K=27$ & 999.636 & 0.0364 & 0.00100313 & 0.3130 & 0.199886 & 0.0570 \\
$K=53$ & 1000.060 & 0.0060 & 0.00100355 & 0.3550 & 0.199830 & 0.0850 \\
\hline
\end{tabular}

Mean Squared Relative Error (RMSRE):

$$
\begin{aligned}
M A R E & =\frac{1}{K} \sum_{i=1}^{K}\left|\frac{N_{i}-\hat{N}_{i}}{N_{i}}\right|, \\
R M S R E & =\sqrt{\frac{1}{K} \sum_{i=1}^{K}\left(\frac{N_{i}-\hat{N}_{i}}{N_{i}}\right)^{2}},
\end{aligned}
$$

where $K, N_{i}$ and $\hat{N}_{i}$ denote the number of data points, the observed values and the estimated values, respectively.

In a large number of numerical experiments it was confirmed that, in terms of the MARE and RMSRE, minimization of the functional $F$ defined by (7) (with data obtained by the centered finite difference approximation method) provides a much better fit than minimization of functional $S$ defined by (4). For example, the results of one experiment with $\sigma^{2}=0.3$ are shown in Table 2 .

Example 3. We are going to fit the Bass model to real diffusion data for seven products: room air conditioners, color televisions, clothes dryers, ultrasound, mammography, foreign language, and accelerated program (Table 3). These data, taken from Mahajan et al. (1986), have been used extensively in the diffusion modeling literature to illustrate the efficiency of estimation procedures (see Mahajan et al., 1986; Schmittlein and Mahajan, 1982; Scitovski and Meler, 2002; Srinivasan and Mason, 1986).

For each product or service, we estimated the unknown parameters $m, p$ and $q$ by minimizing functional $F$ defined by (7), as well as by minimizing functional $S$ defined by (4). For all weights $w_{i}$ we took 1 . Parameter estimates and fit statistics are reported in Tables 4 and 5

As can be seen, in terms of the MARE, minimization of functional $F$ provides a better fit for five products (room air conditioners, color televisions, mammography, foreign language, and accelerated program). In terms of the RMSRE, minimization of the functional $F$ provides a better fit for two products (a foreign language, and an accelerated program).

\section{Conclusions}

The best-known and widely used model in diffusion research is the Bass model. It has three parameters: the coefficient of innovation $(p>0)$, the coefficient of imitation $(q \geq 0)$, and the total market potential $(m>0)$. In practice, the unknown parameters $p, q$ and $m$ are not known in advance and must be estimated from the actual adoption data. There is no unique way to estimate the unknown parameters and many different methods have been proposed in the literature.

In this paper, we have considered the parameter estimation approach for the Bass model based on the nonlinear weighted least squares fitting of the Bass adoption curve. We have shown that the best least squares estimate for the Bass adoption curve does not necessarily exist (Proposition 1). As our main results, we present two theorems (Theorems 1 and 2) on the existence of the least squares estimate, as well as their generalizations in the $l_{s}$ norm $(1 \leq s<\infty)$. Theorem 1 gives a necessary and sufficient condition for the existence of the least squares estimate. For practical purposes, Theorem 2 is extremely important, as it guarantees the existence of the least squares estimate in the case when parameter $m$ is bounded above. Some numerical experiments are included to illustrate the efficiency of our estimation approach.

\section{Acknowledgment}

The authors wish to thank the Ministry of Science, Education and Sports of the Republic of Croatia for funding received through the research grant no. 235-2352818-1034.

\section{References}

Atieg, A. and Watson, G.A. (2004). Use of $l_{p}$ norms in fitting curves and surfaces to data, The ANZIAM Journal 45(E): C187-C200.

Bailey, N.T.J. (1975). The Mathematical Theory of Infectious Diseases and Its Applications, Griffin, London.

Bailey, N.T.J. (1957). The Mathematical Theory of Epidemics, Griffin, London.

Bass, F.M. (1969). A new product growth model for consumer durables, Management Science 15(5): 215-227.

Bates, D.M. and Watts, D.G. (1988). Nonlinear Regression Analysis and Its Applications, Wiley, New York, NY.

Björck, Å. (1996). Numerical Methods for Least Squares Problems, SIAM, Philadelphia, PA.

Demidenko, E.Z. (2008). Criteria for unconstrained global optimization, Journal of Optimization Theory and Applications 136(3): 375-395. 
Table 2. Accuracy of parameter estimates.

\begin{tabular}{|l|c|c|}
\hline & by minimizing $F$ & by minimizing $S$ \\
\hline \hline & $\left(\bar{m}^{\star}, \bar{p}^{\star}, \bar{q}^{\star}\right)=(999.99,0.001005,0.199791)$ & $(\hat{m}, \hat{p}, \hat{q})=(999.94,0.001001,0.199962)$ \\
MARE & 0.008468 & 0.049424 \\
RMSRE & 0.026829 & 0.077858 \\
\hline
\end{tabular}

Table 3. Data listing.

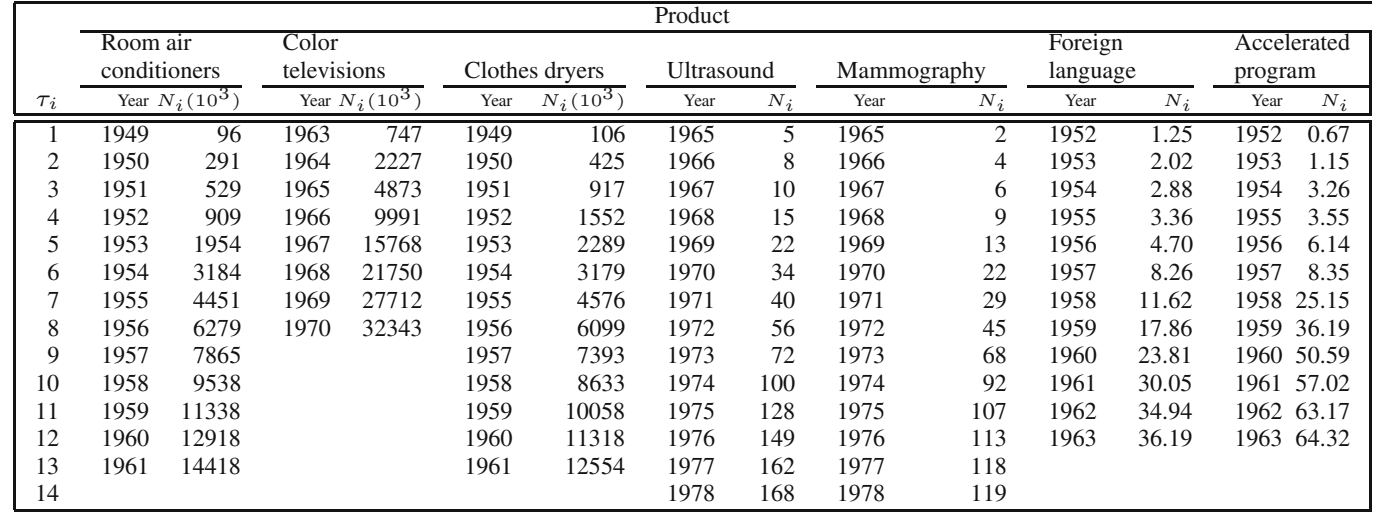

Table 4. Parameter estimates and fit statistics obtained by minimizing functional $F$ (our approach).

\begin{tabular}{|l|r|r|r|r|r|}
\hline Product & $\bar{m}^{\star}$ & $\bar{p}^{\star}$ & $\bar{q}^{\star}$ & MARE & RMSRE \\
\hline \hline Room air conditioners & $18.72 \times 10^{6}$ & 0.00953 & 0.37328 & 0.30810 & 0.50741 \\
Color televisions & $39.69 \times 10^{6}$ & 0.01889 & 0.60920 & 0.10506 & 0.16738 \\
Clothes dryers & $16.50 \times 10^{6}$ & 0.01367 & 0.32565 & 0.19744 & 0.43997 \\
Ultrasound & 167.44 & 0.00136 & 0.61627 & 0.43294 & 0.53512 \\
Mammography & 111.51 & 0.00045 & 0.84864 & 0.43308 & 0.55568 \\
Foreign language & 37.62 & 0.00199 & 0.68890 & 0.35119 & 0.47026 \\
Accelerated program & 64.61 & 0.00084 & 0.90948 & 0.28898 & 0.42592 \\
\hline
\end{tabular}

Table 5. Parameter estimates and fit statistics obtained by minimizing functional $S$ (NLS approach).

\begin{tabular}{|l|r|r|r|r|r|}
\hline Product & $\hat{m}$ & $\hat{p}$ & $\hat{q}$ & MARE & RMSRE \\
\hline \hline Room air conditioners & $18.71 \times 10^{6}$ & 0.00944 & 0.37476 & 0.30984 & 0.47103 \\
Color televisions & $39.66 \times 10^{6}$ & 0.01847 & 0.61586 & 0.11028 & 0.15061 \\
Clothes dryers & $16.50 \times 10^{6}$ & 0.01360 & 0.32670 & 0.19525 & 0.42240 \\
Ultrasound & 167.38 & 0.00132 & 0.62060 & 0.41165 & 0.50388 \\
Mammography & 111.39 & 0.00041 & 0.86065 & 0.44583 & 0.55339 \\
Foreign language & 37.56 & 0.00189 & 0.69676 & 0.40617 & 0.52545 \\
Accelerated program & 64.43 & 0.00074 & 0.92828 & 0.76973 & 1.15919 \\
\hline
\end{tabular}

Demidenko, E.Z. (2006). Criteria for global minimum of sum of squares in nonlinear regression, Computational Statistics \& Data Analysis 51(3): 1739-1753.

Demidenko, E.Z. (1996). On the existence of the least squares estimate in nonlinear growth curve models of exponential type, Communications in Statistics-Theory and Methods 25(1): 159-182.

Dennis, J.E. and Schnabel, R.B. (1996). Numerical Methods for Unconstrained Optimization and Nonlinear Equations, SIAM, Philadelphia, PA.

Gill, P.E., Murray, W. and Wright, M.H. (1981). Practical Optimization, Academic Press, London.
Gonin, R. and Money, A.H. (1989). Nonlinear $L_{p}$-Norm Estimation, Marcel Dekker, New York, NY.

Hadeler, K.P., Jukić, D. and Sabo, K. (2007). Least squares problems for Michaelis Menten kinetics, Mathematical Methods in the Applied Sciences 30(11): 1231-1241.

Jukić, D. (2011). Total least squares fitting Bass diffusion model, Mathematical and Computer Modelling 53(9-10): 1756-1770.

Jukić, D. (2013) On nonlinear weighted least squares estimation of Bass diffusion model, Applied Mathematics and Computation, (accepted).

Jukić, D. and Marković, D. (2010). On nonlinear weighted errors-in-variables parameter estimation problem in the 
three-parameter Weibull model, Applied Mathematics and Computation 215(10): 3599-3609.

Jukić, D. (2009). On the existence of the best discrete approximation in $l_{p}$ norm by reciprocals of real polynomials, Journal of Approximation Theory 156(2): 212-222.

Jukić, D., Benšić, M. and Scitovski, R. (2008). On the existence of the nonlinear weighted least squares estimate for a three-parameter Weibull distribution, Computational Statistics \& Data Analysis 52(9): 4502-4511.

Jukić, D., Kralik, G. and Scitovski, R. (2004). Least squares fitting Gompertz curve, Journal of Computational and Applied Mathematics 169(2): 359-375.

Mahajan, V. Muller, E. and Wind, Y. (Eds.). (2000). NewProduct Diffusion Models, Kluwer Academic Publishers, London.

Mahajan, V., Mason, C.H. and Srinivasan, V. (1986). An evaluation of estimation procedures for new product diffusion models, in V. Mahajan and Y. Wind (Eds.), Innovation Diffusion Models of New Product Acceptance, Ballinger Publishing Company, Cambridge, pp. 203-232.

Mahajan, V. and Sharma, S. (1986). A simple algebraic estimation procedure for innovation diffusion models of new product acceptance, Technological Forecasting and Social Change 30(4): 331-346.

Marković, D. and Jukić, D. (2010). On nonlinear weighted total least squares parameter estimation problem for the three-parameter Weibull density, Applied Mathematical Modelling 34(7): 1839-1848.

Marković, D., Jukić, D. and Benšić, M. (2009). Nonlinear weighted least squares estimation of a three-parameter Weibull density with a nonparametric start, Journal of Computational and Applied Mathematics 228(1): 304-312.

Rogers, E.M. (1962). Diffusion of Innovations, The Free Press, New York, NY.

Ross, G.J.S. (1990). Nonlinear Estimation, Springer, New York, NY.
Schmittlein, D. and Mahajan, V. (1982). Maximum likelihood estimation for an innovation diffusion model of new product acceptance, Marketing Science 1(1): 57-78.

Scitovski, R. and Meler, M. (2002). Solving parameter estimation problem in new product diffusion models, $A p$ plied Mathematics and Computation 127(1): 45-63.

Seber, G.A.F. and Wild, C.J. (1989). Nonlinear Regression, Wiley, New York, NY.

Srinivasan, V. and Mason, C.H. (1986). Nonlinear least squares estimation of new product diffusion models, Marketing Science 5(2): 169-178.

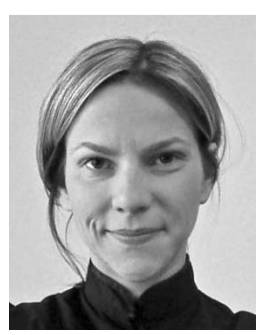

Darija Marković holds a B.Sc. degree in mathematics and computer science from the University of Osijek, as well as an M.Sc. in mathematics from the University of Zagreb. In 2009 she obtained a Ph.D. in mathematics from the University of Zagreb. Presently she is an assistant professor at the Department of Mathematics, University of Osijek. Her research interests cover applied and numerical mathematics, specifically parameter estimation, mathematical modelling, nonlinear least squares and smoothing methods. She has co-authored several papers in journals and conference proceedings on these subjects.

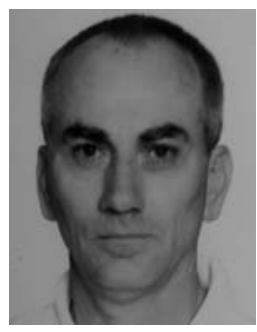

Dragan Jukić received a B.Sc. degree in mathematics and physics from the University of Osijek, Croatia, in 1986, as well as an M.Sc. and a Ph.D. degree in mathematics from the University of $\mathrm{Za}$ greb, Croatia, in 1990 and 1996, respectively. In 2000 he became a professor and in 2004 a full professor at the Department of Mathematics, University of Osijek. His current research interests include mathematical modelling, parameter estimation, curve fitting, nonlinear least squares and smoothing methods. He is the author or coauthor of more than 50 journal and conference papers.

Received: 7 February 2012 Revised: 6 August 2012 